\title{
School Bullying: Cause or Consequence?
}

\author{
Rajesh Sagar $^{1}$ (1) $\cdot$ Mahadev Singh Sen ${ }^{1}$
}

Received: 29 July 2021 / Accepted: 5 August 2021 / Published online: 16 August 2021

(c) Dr. K C Chaudhuri Foundation 2021

We read the article by Malhi and Bharti with great interest. The study explores the relationship of victimized children with somatic symptoms, which is a less studied yet very important aspect. The paper concludes that the prevalence of bullying is high and needs urgent attention; also, that the burden of the physical complaints in the victims is high. Selfreported adjustment with teachers was found to be an important factor associated with physical symptoms [1]. This might be a proxy marker for the lack of conducive environment in school setting where a child can express his negative experience, and thus for having internalizing symptoms. Therefore, it becomes even more important for the stakeholders to develop and implement novel and culturally sensitive strategies to counter bullying at school. These strategies should have an active involvement of the teachers which should also involve increasing their sensitivity to the situation.

An important issue in research on bullying is establishing the causal relationship, i.e., is it the cause or the consequence. Children with physical and psychological problems tend to be vulnerable to bullying and, at the same time, having somatic complaints. Thus, ruling out such issues becomes important to establish the significance of the relationship with somatic symptoms independently. Previous studies have already concluded significant association of physical symptoms with psychological factors like symptoms of anxiety and depression [2]. Many such signs and symptoms may just be a normal variation in general population, and thus, hold no clinical significance. To tackle this, one approach could have been to take the already collected variables, i.e., child's academic performance and attendance into account as a proxy marker for disability or dysfunction. This cannot substitute the face-to-face in depth interviews but can approximate 'clinical significance criterion' in Diagnostic and Statistical Manual of Mental Disorders (DSM) or 'clinically

Rajesh Sagar

rsagar29@gmail.com

1 Department of Psychiatry, All India Institute of Medical Sciences, Ansari Nagar, New Delhi 110029, India recognizable symptoms' in International Classification of Diseases (ICD) [3]. Thus, analyzing and comparing these variables with other variables in the study would be helpful in establishing the significance of physical symptoms in clinical context and the impact of bullying in the child's life.

Another aspect which was not clear in the study was the translation and validation of the California Bullying Victimization Scale (CBVS) in local context. Identifying an old tool in new light in terms of linguistic equivalence and conceptual equivalence while preserving the cultural context of the second language is very important, especially in a self-reporting scale. Further, looking into the relationship between the variables of the study whilst keeping in mind the psychometric properties of the translation of the scale are important when we interpret the results and apply to the real world scenario [4].

Lastly, we would like to state that the concept and dimensions of school bullying have evolved in the past few decades and the research is still evolving. The segregation of social, regional, or cultural bullying against personal bullying is another factor which could be explored in future studies [5]. This is especially relevant in the context of Indian population with diverse social, religious, and cultural backgrounds. Many authors have highlighted this aspect and its importance in victimization and developing psychiatric issues related to bullying $[5,6]$. This would contribute further to understand bullying and develop targeted antibullying interventions.

\section{Declarations}

Conflict of Interest None.

\section{References}

1. Malhi P, Bharti B. School bullying and association with somatic complaints in victimized children. Indian J Pediatr. 2021. https:// doi.org/10.1007/s12098-020-03620-5. 
2. Ali A, Borah S. Assessment of somatic symptoms among school going children: A study from north east India. Indian J Health Wellbeing. 2014;5:354-7.

3. Sagar R, Chawla N, Sen MS. Is it correct to estimate mental disorder through online surveys during COVID-19 pandemic? Psychiatry Res. 2020;291:113251.

4. Kozanhan B, Yildiz M. Questionnaire translation and questionnaire validation are not the same. Int J Obstet Anesth. 2021;45:165.

5. Rodríguez-Hidalgo AJ, Calmaestra J, Casas JA, Ortega-Ruiz R. Ethnic-cultural bullying versus personal bullying: specificity and measurement of discriminatory aggression and victimization among adolescents. Front Psychol. 2019;10:46.

6. Atwal K, Wang C. Religious head covering, being perceived as foreigners, victimization, and adjustment among Sikh American adolescents. Sch Psychol. 2019;34:233-43.

Publisher's Note Springer Nature remains neutral with regard to jurisdictional claims in published maps and institutional affiliations. 